\title{
gु
}

\section{Evidence of Halo Assembly Bias in Massive Clusters}

\author{
Hironao Miyatake, ${ }^{1,2,3,{ }^{*}}$ Surhud More, ${ }^{2}$ Masahiro Takada, ${ }^{2}$ David N. Spergel, ${ }^{1,2}$ Rachel Mandelbaum, ${ }^{4}$ \\ Eli S. Rykoff, ${ }^{5,6}$ and Eduardo Rozo ${ }^{7}$ \\ ${ }^{1}$ Department of Astrophysical Sciences, Princeton University, Peyton Hall, Princeton New Jersey 08544, USA \\ ${ }^{2}$ Kavli Institute for the Physics and Mathematics of the Universe (WPI), UTIAS, The University of Tokyo, Chiba 277-8583, Japan \\ ${ }_{3}^{3}$ Jet Propulsion Laboratory, California Institute of Technology, Pasadena, California 91109, USA \\ ${ }^{4}$ Department of Physics, McWilliams Center for Cosmology, Carnegie Mellon University, Pittsburgh, Pennsylvania 15213, USA \\ ${ }^{5}$ Kavli Institute for Particle Astrophysics \& Cosmology, P. O. Box 2450, Stanford University, Stanford, California 94305, USA \\ ${ }^{6}$ SLAC National Accelerator Laboratory, Menlo Park, California 94025, USA \\ ${ }^{7}$ Department of Physics, University of Arizona, 1118 E 4th St, Tucson, Arizona 85721, USA
}

(Received 30 June 2015; revised manuscript received 2 November 2015; published 25 January 2016)

\begin{abstract}
We present significant evidence of halo assembly bias for SDSS redMaPPer galaxy clusters in the redshift range [0.1, 0.33]. By dividing the 8,648 clusters into two subsamples based on the average member galaxy separation from the cluster center, we first show that the two subsamples have very similar halo mass of $M_{200 m} \simeq 1.9 \times 10^{14} h^{-1} M_{\odot}$ based on the weak lensing signals at small radii $R \lesssim 10 h^{-1} \mathrm{Mpc}$. However, their halo bias inferred from both the large-scale weak lensing and the projected autocorrelation functions differs by a factor of $\sim 1.5$, which is a signature of assembly bias. The same bias hypothesis for the two subsamples is excluded at $2.5 \sigma$ in the weak lensing and $4.4 \sigma$ in the autocorrelation data, respectively. This result could bring a significant impact on both galaxy evolution and precision cosmology.
\end{abstract}

DOI: 10.1103/PhysRevLett.116.041301

Since massive cluster-sized halos of cold dark matter (CDM) emerge from the rarest peaks in the primordial Gaussian random density field [1,2], their clustering amplitudes at large scales is highly biased compared to the underlying mass distribution [3-6]. In the standard version of the halo model, the clustering amplitude depends only on halo mass (see, e.g., Ref. [7]).

Do secondary parameters other than the halo mass affect the clustering amplitude? Do observations of galaxy clusters reveal halo assembly bias, an effect predicted both by analytical theory and simulations [8-12]?

In this Letter, we use a combination of the clustering and the weak gravitational lensing of clusters and present the first significant evidence of a difference in the large scale bias for cluster samples of the same mass. We divide our sample of galaxy clusters into two based on the average projected radial separation of member galaxies, and investigate the difference in their clustering amplitude on large scales. In Ref. [13], we will show that the two cluster subsamples have different mass accretion rates and hence different assembly histories, confirming these observations to be strong evidence for halo assembly bias. The distinguishing feature of our analysis is to use weak lensing to verify that the subsamples have similar halo masses, but different halo biases. There have been several claims for the evidence of assembly bias on galaxy scales (e.g., Ref. [14-16]). However, Ref. [17] found that the difference in clustering properties could all be explained as due to difference in halo mass or contamination by satellite galaxies and concluded that there was no significant evidence of the assembly bias for galaxy-scale halos.
Throughout this Letter, we adopt a flat $\Lambda \mathrm{CDM}$ cosmological model with matter density parameter $\Omega_{m}=0.27$ and the Hubble parameter $h=0.7$.

Cluster subsamples.-We use the publicly available catalog of galaxy clusters identified from the SDSS DR8 photometric galaxy catalog by the red-sequence Matchedfilter Probabilistic Percolation (redMaPPer) cluster finding algorithm (v5.10 at Ref. [18], also see Refs. [19,20] for details). redMaPPer uses the ugriz magnitudes and their errors, to group spatial concentrations of red-sequence galaxies at similar redshifts into clusters. For each cluster, the catalog contains an optical richness estimate $\lambda$, a photometric redshift estimate $z_{\lambda}$, as well as the position and probabilities of five candidate central galaxies $p_{\text {cen }}$ [19]. A separate member galaxy catalog provides a list of members for each cluster, each of which is assigned a membership probability, $p_{\text {mem }}[19]$.

We use a sample of clusters with $20<\lambda<100$ and $0.1 \leq z_{\lambda} \leq 0.33$. The richness cuts ensure a pure and statistically meaningful sample of clusters at all richness bins, while the redshift cuts select a nearly volume-limited sample of clusters [19], resulting in a sample of 8,648 clusters. For the weak lensing and clustering measurements, we use 100 times as many random points as real clusters, incorporating the survey geometry, depth variations, and distributions of cluster redshift and richness (see Refs. [21,22] for details on the use of random catalogs).

As a proxy for the assembly history of the clusters, we use the average projected separation of member galaxies from the cluster center, $\left\langle R_{\mathrm{mem}}\right\rangle$. For each cluster, we compute 


$$
\left\langle R_{\mathrm{mem}}\right\rangle=\sum_{i} p_{\mathrm{mem}, i} R_{\mathrm{mem}, i} / \sum_{i} p_{\mathrm{mem}, i}
$$

where the summation runs over all member galaxies, and $R_{i}$ is the projected separation of the $i$ th member from the cluster center. Throughout this Letter we use the position of the most probable central galaxy in each cluster region as a proxy of the cluster center. We employ 14 equally spaced bins both in redshift and $\lambda$ and obtain a spline fit for the median of $\left\langle R_{\mathrm{mem}}\right\rangle$ as a function of redshift and richness. We then define the two subsamples by the upper and lower halves of clusters in each bin of richness and redshift space [23]. The ratio of $\left\langle R_{\mathrm{mem}}\right\rangle$ for a bootstrapped realization of galaxy cluster pairs from the large- and the small- $\left\langle R_{\text {mem }}\right\rangle$ samples selected within the same richness and redshift has a distribution with median $1.18_{-0.09}^{+0.14}$ (the error bars correspond to the 16th and 84th percentile). The large- and small- $\left\langle R_{\text {mem }}\right\rangle$ subsamples consist of 4,235 and 4,413 clusters, respectively. By construction, the two subsamples have almost identical distributions of redshift and richness.

Weak lensing measurements.-The weak gravitational lensing (WL) effect on the shapes of background galaxies can be used to measure the average mass distribution around galaxy clusters. We use the shape catalog of Ref. [24], which is based on the photometric galaxy catalog from the SDSS DR8 for this purpose. The galaxy shapes are measured by the re-Gaussianization technique [25], and the systematic uncertainties involved in the shape measurements have been investigated in great detail in Ref. [21]. The redshifts of source galaxies are estimated based on the photo- $z$ code ZEBRA [26,27]. The accuracy of the photometric redshift is not crucial for our study, because the populations of source galaxies used to compare the WL signals of our two cluster subsamples are identical. To measure the cluster WL profiles, we use the same method as described in Refs. [22,28].

The top and middle panels of Fig. 1 show $\Delta \Sigma(R)$, the excess surface mass density at a given projected radius $R$ [21] for the large- and small- $\left\langle R_{\text {mem }}\right\rangle$ subsamples of clusters, respectively. The covariance matrix for each of the measurements was estimated based on 83 jackknife regions of approximately equal area covering the SDSS footprints (63 and 20 for the northern and southern hemisphere footprints, respectively). The figure shows that the WL signals of the two subsamples have very similar amplitudes at small radii, $R \lesssim 10 h^{-1} \mathrm{Mpc}$, and consequently similar average halo masses. However, the WL signals on larger scales, $10 \lesssim R /\left[h^{-1} \mathrm{Mpc}\right] \leq 50$, display a significant difference, a signature of assembly bias, as explicitly shown in the bottom panel.

We perform halo model fits to the measurements of the WL signal of each cluster subsample. Following Ref. [29], we employ a simple six parameter model fit to the WL signal,

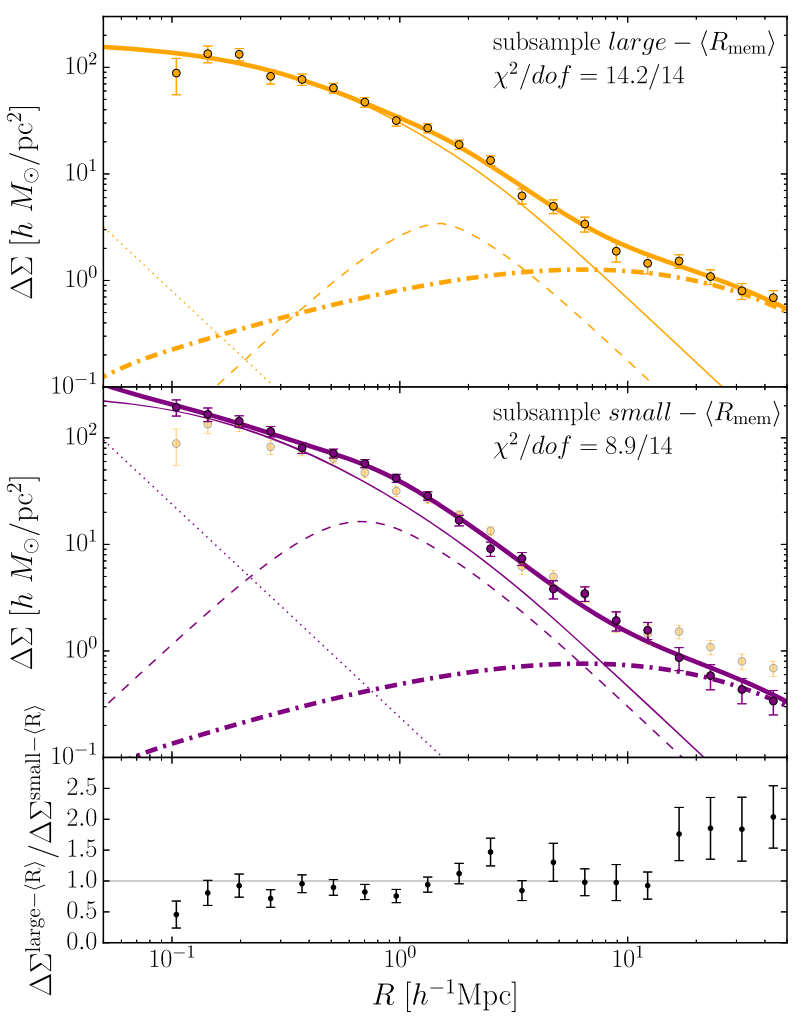

FIG. 1. Halo mass consistency and assembly bias from the WL signal. The data points with error bars in the top and middle panels show the excess surface mass density profile as a function of the cluster-centric projected radius (in comoving units), obtained from the WL measurements for the large- and small- $\left\langle R_{\text {mem }}\right\rangle$ subsamples of redMaPPer clusters [see Eq. (1)], respectively. The points from the top panel are reproduced in semitransparent color in the middle panel for comparison. The mass profiles at small radii $R \lesssim 10 h^{-1} \mathrm{Mpc}$ appear to have similar amplitudes with slightly different shapes, but show a difference in amplitude at $R \gtrsim 10 h^{-1} \mathrm{Mpc}$, as expected from assembly bias. The bold solid line shows the best-fit halo model, the thin solid line is the centered 1-halo term, the dashed line is the off-centered 1-halo term, while the dotted line corresponds to the stellar mass contribution from the central galaxy. Comparison between the dot-dashed lines in the two panels implies that the 2-halo term contributions, which arise from the average mass distribution surrounding the clusters, are different by a factor of 1.6. The bottom panel shows the ratios of the lensing signals, highlighting a clear deviation from unity at $R \gtrsim 15 \mathrm{Mpc} / h$.

$$
\begin{aligned}
\Delta \Sigma( & \left.R ; M_{200 m}, c_{200 m}, q_{\mathrm{cen}}, \alpha_{\mathrm{off}}, M_{*}, b\right) \\
= & q_{\mathrm{cen}} \Delta \Sigma^{\mathrm{NFW}}\left(R ; M_{200 m}, c_{200 m}\right) \\
& +\left(1-q_{\mathrm{cen}}\right) \Delta \Sigma^{\mathrm{NFW}, \mathrm{off}}\left(R ; M_{200 m}, c_{200 m}, \alpha_{\mathrm{off}}\right) \\
& +\Delta \Sigma^{*}\left(R ; M_{*}\right)+\Delta \Sigma^{2 \text {-halo }}(R ; b) .
\end{aligned}
$$

The first term corresponds to the halo mass profile for the fraction $q_{\text {cen }}$ of clusters whose centers have been correctly identified, while the second term corresponds to the clusters with misidentified centers. We assume that the halo mass profile is a smoothly truncated version of 
the Navarro-Frenk-White (NFW) profile [30], proposed in Ref. [31], specified by the halo mass and concentration parameter, $M_{200 m}$ and $c_{200 m}$ [32]. We adopt $\tau_{200 m}=2.6$ for the smoothing kernel, although we confirmed that our result of similar masses for the two subsamples is not sensitive to the chosen value of $\tau_{200 m}$. We simply consider a single mass bin for host halos. We assume that the normalized profile of the positions of off-centered clusters with respect to their true center is given by $u_{\text {off }}(r) \propto \exp \left[-r^{2} /\left(2 \alpha_{\text {off }}^{2} R_{200 m}^{2}\right)\right]$, where $\alpha_{\text {off }}$ describes the ratio of the off-centering radius to $R_{200 m}$. We also truncate the off-centering profile to zero at $r>R_{200 m}$. The third term, $\Delta \Sigma_{*} \equiv M_{*} /\left(\pi R^{2}\right)$, models a possible stellar mass contribution from the central galaxies assuming a point mass. The fourth term $\Delta \Sigma^{2 \text {-halo }}(R)$ models the lensing contribution arising from the two-point correlation function between the clusters and the surrounding mass distribution. We employ the model given as $\Delta \Sigma^{2 \mathrm{~h}}(R)=$ $b \int(k \mathrm{~d} k / 2 \pi) \bar{\rho}_{m} P_{m}^{L}\left(k ; z_{\mathrm{cl}}\right) J_{2}(k R)$, where $\bar{\rho}_{m}$ is the mean mass density today, $b$ is the linear bias parameter, and $P_{m}^{L}\left(k ; z_{\mathrm{cl}}\right)$ is the linear mass power spectrum at the averaged cluster redshift $z_{\mathrm{cl}}=0.24$, for the $\Lambda \mathrm{CDM}$ model.

We explore the posterior distribution of the parameters given our measurements using the Markov Chain Monte Carlo (MCMC) technique [33]. We use flat priors for all the parameters: $M_{200 m} /\left[10^{14} h^{-1} M_{\odot}\right] \in$ $[0.5,50], \quad c_{200 m} \in[1,10], \quad q \in[0,1], \quad \alpha_{\text {off }} \in\left[10^{-4}, 1\right], \quad M_{*} /$ $\left[10^{12} h^{-1} M_{\odot}\right] \in[0,10]$, and $b \in[0,10]$. In Fig. 2, we show the posterior distributions of the parameters $M_{200 m}, c_{200 m}$ as well as $b$, comparing results for the small- and large- $\left\langle R_{\text {mem }}\right\rangle$ subsamples, after marginalization over the off-centering parameters and the stellar mass contribution [34]. The halo masses are consistent with each other within the error bars: $M_{200 m} /\left[10^{14} h^{-1} M_{\odot}\right]=1.87_{-0.14}^{+0.12}$ or $1.88_{-0.18}^{+0.16}$ for the small- and large- $\left\langle R_{\mathrm{mem}}\right\rangle$ subsamples, respectively. The concentration parameters have strong degeneracies with the off-centering parameters, but turn out to be similar for the two subsamples after the marginalization. The halo bias parameters are $b=2.17 \pm 0.31$ and $3.67_{-0.37}^{+0.40}$, respectively. The ratio $b^{\text {large }-\left\langle R_{\text {mem }}\right\rangle} / b^{\text {small }-\left\langle R_{\text {mem }}\right\rangle}=1.64_{-0.26}^{+0.31}$, a $2.5 \sigma$ deviation from unity. For comparison, even if we take the halo masses for the two subsamples at the extreme ends of their posterior distributions within their 95\% C.L. interval, the halo bias model of Ref. [6] predicts that the ratio is at most 1.13 .

Clustering measurements.-We now consider the autocorrelation functions of clusters in the two subsamples to further confirm the difference in the large-scale bias in the WL signals. To avoid redshift-space distortions, we consider the projected correlation function

$$
w_{p}(R)=2 \int_{0}^{\Pi_{\max }=100 h^{-1} \mathrm{Mpc}} d \Pi \xi(R, \Pi),
$$

where $R$ and $\Pi$ are the projected and line-of-sight separations between cluster pairs, and $\xi(R, \Pi)$ is the

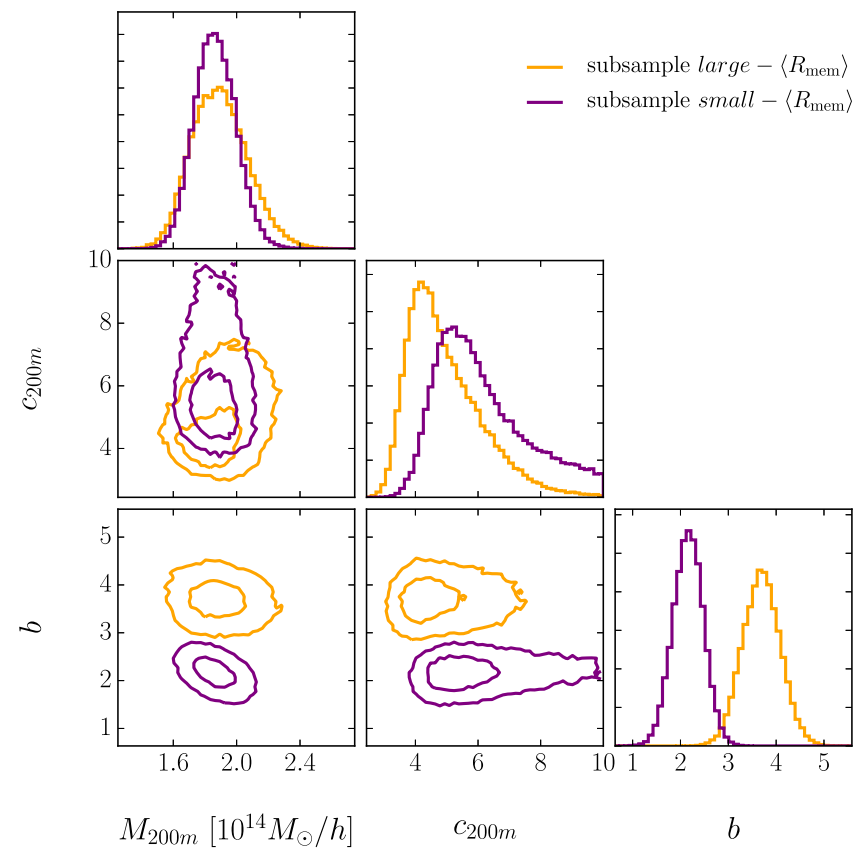

FIG. 2. The posterior distributions of halo model parameters given the WL signals for each of the two cluster subsamples shown in Fig. 1. The distributions include marginalization over nuisance parameters which correspond to off-centering effects and stellar mass contribution from the central galaxy (see text for details). The parameters $M_{200 m}$ and $c_{200 m}$ are the halo mass and concentration parameters that specify the average NFW profile of the clusters (1-halo term), while $b$ is the linear halo bias of the cluster subsample. The posterior distributions show the large- and small- $\left\langle R_{\text {mem }}\right\rangle$ subsamples have similar halo mass, but display a significant difference in their bias parameters.

three-dimensional correlation function. We compute the three-dimensional correlation function $\xi(R, \Pi)$ using the Landy and Szalay estimator [35], and replace the integral over the line of sight by discrete summation with $\Delta \Pi=1 h^{-1} \mathrm{Mpc}$.

In Fig. 3, we show the ratio of the projected autocorrelation functions measured from our subsamples, relative to that of the parent sample (i.e., all the clusters), along with jackknife error estimates. Over the range of separations we have considered, the two subsamples show significantly different clustering amplitudes than the parent sample, giving an independent confirmation of assembly bias. To quantify the significance, we fit a constant parameter model, $\zeta_{0}$, to $\zeta(R)=\sqrt{w_{p}^{\text {large }-\left\langle R_{\mathrm{mem}}\right\rangle} / w_{p}^{\text {small- }\left\langle R_{\mathrm{mem}}\right\rangle}}$, accounting for the covariance between the data points. We obtain $\zeta_{0}=1.40 \pm 0.09$ (1.34 \pm 0.12 if we restrict to $R>10 h^{-1} \mathrm{Mpc}$ ), corresponding to a $4.4 \sigma$ deviation from $\zeta_{0}=1$. Using both the ratios of the clustering signal and the lensing signals at the three outermost bins in Fig. 1, we obtain $\zeta_{0}=1.41 \pm 0.09$ with the significance of $4.7 \sigma$, after accounting for the cross-covariance between the clustering and lensing signals. To be explained by halo mass alone, the bias ratio of 1.4 (1.6) implied from the clustering 


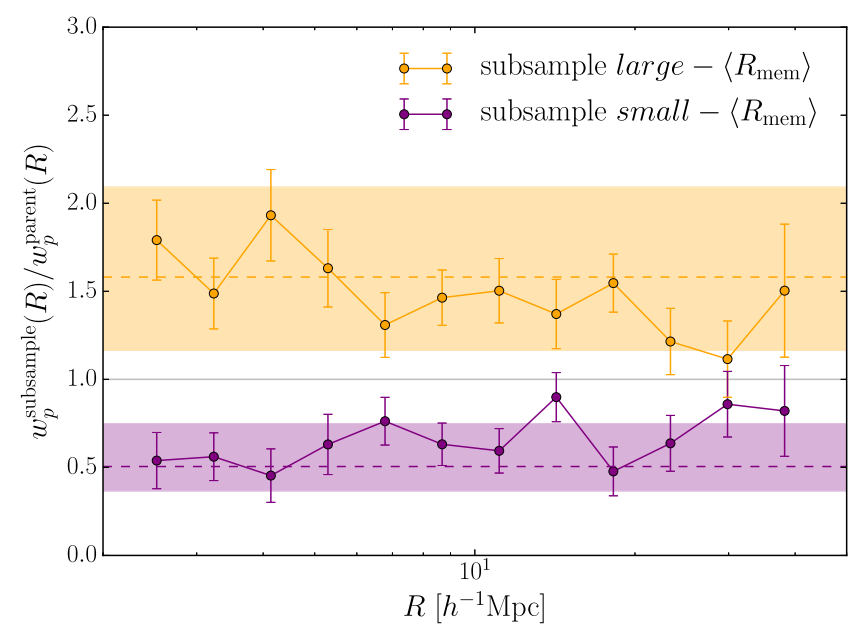

FIG. 3. Halo assembly bias from the projected clustering signal: The projected autocorrelation function of clusters for each of the large- and small- $\left\langle R_{\text {mem }}\right\rangle$ subsamples, relative to that of the full sample (i.e., all clusters). The clustering signals at large separations, $R \gtrsim 2 h^{-1} \mathrm{Mpc}$, show a significant difference, which is consistent with the WL measurements in Fig. 1 as shown by the shaded regions.

(lensing) measurements requires a factor of 3 (4) difference in the halo masses, which is disfavored by the 1-halo lensing signals.

The shaded regions in Fig. 3 show that the difference from the clustering amplitudes is consistent with the WL measurements, within the error bars. The shaded regions were obtained with the results of fits to the lensing signals of the subsamples and the full sample as in Fig. 2. They show the posterior distribution of the square of bias ratio of each subsample to the full sample, after marginalization over other parameters. The dashed lines correspond to the value of the maximum posterior probability.

Conclusion and discussion.-A combination of WL and clustering measurements of redMaPPer galaxy clusters show evidence of the assembly bias for cluster-sized halos with mass $\sim 2 \times 10^{14} h^{-1} M_{\odot}$, with the significance level of $2.5 \sigma$ and $4.4 \sigma$ for each of two observables. One of the implications of this measurement is that the halo mass based on large-scale clustering amplitude is sensitive to the cluster selection function, which should be taken into account for precision cosmology.

Could the large difference in halo bias arise from orientation biases in the cluster identification algorithm? Could the small- $\left\langle R_{\text {mem }}\right\rangle$ clusters preferentially reside in smaller mass halos and consequently have smaller bias, but their weak lensing mass is large due to a filament aligned along the line of sight? For the halo bias difference to arise just from a halo mass difference, the subsamples have to differ in halo mass by a factor of $\sim 3-4$, which is quite unlikely to occur just by projection effects (see, e.g., Ref. [36,37]). Clusters affected by projection (intrinsically lower mass systems) are expected to cause an increase, contrary to a decrease in $\left\langle R_{\text {mem }}\right\rangle$ [38]. We have explicitly verified that the line-of-sight velocity dispersions of member galaxies around the two cluster subsamples are very similar as well. Furthermore, our preliminary investigations indicate that a number of properties of central galaxies (such as their stellar masses, stacked spectra, velocity dispersions) in the two subsamples also do not show any significant differences.

It is worth exploring whether the amplitude of the observed assembly bias is consistent with predictions in $\Lambda$ CDM cosmologies. Reference [10] used $N$-body simulations to study the halo assembly bias in $\Lambda$ CDM models, and found that the bias difference for rare objects such as our clusters, when subdivided by the halo concentration, is $\sim 1.25$, somewhat smaller than our finding (difference of $1.40 \pm 0.09$ for the clustering measurement). However, there are differences in our method: we used the distribution of member galaxies (which should correspond to subhalo locations) for the subdivision, the lensing measurements do not show a clear difference in the halo concentration for the two subsamples (Fig. 2), and the scales we consider include the mildly nonlinear regime. Our preliminary analysis using a high-resolution $N$-body simulation, indicates qualitative agreement between the sign and strength of the signal in the simulations and in the data. These results will be presented elsewhere [13].

A proper comparison between data and simulations will require a careful study of the cluster selection function, as well as observational effects due to line-of-sight projections and the photo- $z$ uncertainties, which can be studied by running the same cluster-finding algorithm on realistic simulated galaxy catalogs with full halo assembly histories. Additionally, on the observational side, one could improve the signal-to-noise ratio of the measurement by using the cross-correlations of the cluster samples of this study with a sample of galaxies such as a catalog of luminous red galaxies that has a much higher spatial number density. We defer these topics to a study in the near future.

We thank Tom Abel, Neal Dalal, Oliver Hahn, Benedikt Diemer, and Andrey Kravtsov for enlightening discussions. We also thank the anonymous referees for a careful reading of the manuscript and their suggestions. H. M. is supported in part by Japan Society for the Promotion of Science (JSPS) Research Fellowships for Young Scientists and by the Jet Propulsion Laboratory, California Institute of Technology, under a contract with the National Aeronautics and Space Administration. M. T. and S. M. are supported by World Premier International Research Center Initiative (WPI Initiative), MEXT, Japan, and by the FIRST program "Subaru Measurements of Images and Redshifts (SuMIRe)," CSTP, Japan. S. M. and M. T. are also supported by Grant-inAid for Scientific Research from the JSPS Promotion of Science (No. 15K17600, No. 23340061, and No. 26610058), MEXT Grant-in-Aid for Scientific Research on Innovative Areas (No. 15H05893) and by JSPS Program for Advancing Strategic International Networks to Accelerate the 
Circulation of Talented Researchers. D. N. S. is partially supported by the NSF AST-1311756 and NASA NNX14AH67G. R. M. acknowledges the support of the Department of Energy Early Career Award program. E. S. R. is partially supported by the U.S. Department of Energy contract to SLAC No. DE-AC02-76SF00515. Funding for SDSS-III has been provided by the Alfred P. Sloan Foundation, the Participating Institutions, the National Science Foundation, and the U.S. Department of Energy Office of Science. SDSS-III is managed by the Astrophysical Research Consortium for the Participating Institutions of the SDSS-III Collaboration including the University of Arizona, the Brazilian Participation Group, Brookhaven National Laboratory, Carnegie Mellon University, University of Florida, the French Participation Group, the German Participation Group, Harvard University, the Instituto de Astrofisica de Canarias, the Michigan State/Notre Dame/ JINA Participation Group, Johns Hopkins University, Lawrence Berkeley National Laboratory, Max Planck Institute for Astrophysics, Max Planck Institute for Extraterrestrial Physics, New Mexico State University, New York University, Ohio State University, Pennsylvania State University, University of Portsmouth, Princeton University, the Spanish Participation Group, University of Tokyo, University of Utah, Vanderbilt University, University of Virginia, University of Washington, and Yale University.

*Hironao.Miyatake@jpl.nasa.gov

[1] W. H. Press and P. Schechter, Astrophys. J. 187, 425 (1974).

[2] A. V. Kravtsov and S. Borgani, Annu. Rev. Astron. Astrophys. 50, 353 (2012).

[3] N. Kaiser, Astrophys. J. 284, L9 (1984).

[4] H. J. Mo and S. D. M. White, Mon. Not. R. Astron. Soc. 282, 347 (1996).

[5] R. K. Sheth, H. J. Mo, and G. Tormen, Mon. Not. R. Astron. Soc. 323, 1 (2001).

[6] J. L. Tinker, B. E. Robertson, A. V. Kravtsov, A. Klypin, M. S. Warren, G. Yepes, and S. Gottlöber, Astrophys. J. 724, 878 (2010).

[7] A. Cooray and R. Sheth, Phys. Rep. 372, 1 (2002).

[8] R. K. Sheth and G. Tormen, Mon. Not. R. Astron. Soc. 350, 1385 (2004).

[9] L. Gao, V. Springel, and S. D. M. White, Mon. Not. R. Astron. Soc. 363, L66 (2005); L. Gao and S. D. M. White, Mon. Not. R. Astron. Soc. 377, L5 (2007).

[10] R. H. Wechsler, A. R. Zentner, J. S. Bullock, A. V. Kravtsov, and B. Allgood, Astrophys. J. 652, 71 (2006).

[11] Y. Li, H. J. Mo, and L. Gao, Mon. Not. R. Astron. Soc. 389, 1419 (2008)

[12] N. Dalal, M. White, J. R. Bond, and A. Shirokov, Astrophys. J. 687, 12 (2008).

[13] S. More et al. (to be published).

[14] X. Yang, H. J. Mo, and F. C. van den Bosch, Astrophys. J. Lett. 638, L55 (2006).
[15] J. L. Tinker, M. R. George, A. Leauthaud, K. Bundy, A. Finoguenov, R. Massey, J. Rhodes, and R. H. Wechsler, Astrophys. J. Lett. 755, L5 (2012).

[16] A. P. Hearin, D. F. Watson, and F. C. van den Bosch, Mon. Not. R. Astron. Soc. 452, 1958 (2015).

[17] Y.-T. Lin, R. Mandelbaum, Y.-H. Huang, H.-J. Huang, N. Dalal, B. Diemer, H.-Y. Jian, and A. Kravtsov, arXiv:1504.07632.

[18] http://risa.stanford.edu/redmapper/.

[19] E. S. Rykoff et al., Astrophys. J. 785, 104 (2014).

[20] E. Rozo, E. S. Rykoff, M. Becker, R. M. Reddick, and R. H. Wechsler, Mon. Not. R. Astron. Soc. 453, 38 (2015).

[21] R. Mandelbaum, C. M. Hirata, U. Seljak, J. Guzik, N. Padmanabhan, C. Blake, M. R. Blanton, R. Lupton, and J. Brinkmann, Mon. Not. R. Astron. Soc. 361, 1287 (2005).

[22] H. Miyatake, S. More, R. Mandelbaum, M. Takada, D. N. Spergel, J.-P. Kneib, D. P. Schneider, J. Brinkmann, and J. R. Brownstein, Astrophys. J. 806, 1 (2015).

[23] See Supplemental Material at http://link.aps.org/ supplemental/10.1103/PhysRevLett.116.041301 for the figure for the definitions of the two subsamples in the redshift and richness plane.

[24] R. Reyes, R. Mandelbaum, J. E. Gunn, R. Nakajima, U. Seljak, and C. M. Hirata, Mon. Not. R. Astron. Soc. 425, 2610 (2012).

[25] C. Hirata and U. Seljak, Mon. Not. R. Astron. Soc. 343, 459 (2003).

[26] R. Feldmann et al., Mon. Not. R. Astron. Soc. 372, 565 (2006).

[27] R. Nakajima, R. Mandelbaum, U. Seljak, J. D. Cohn, R. Reyes, and R. Cool, Mon. Not. R. Astron. Soc. 420, 3240 (2012).

[28] R. Mandelbaum, A. Slosar, T. Baldauf, U. Seljak, C. M. Hirata, R. Nakajima, R. Reyes, and R. E. Smith, Mon. Not. R. Astron. Soc. 432, 1544 (2013).

[29] C. Hikage, R. Mandelbaum, M. Takada, and D. N. Spergel, Mon. Not. R. Astron. Soc. 435, 2345 (2013).

[30] J. F. Navarro, C. S. Frenk, and S. D. M. White, Astrophys. J. 462, 563 (1996).

[31] M. Oguri and T. Hamana, Mon. Not. R. Astron. Soc. 414, 1851 (2011).

[32] Throughout this Letter we employ the halo mass and concentration definitions defined for the 200 overdensity with respect to the mean mass density.

[33] D. Foreman-Mackey, D. W. Hogg, D. Lang, and J. Goodman, Publ. Astron. Soc. Pac. 125, 306 (2013).

[34] The off-centering parameters and the stellar mass are very weakly constrained, e.g., at $68 \%$ confidence, $q_{\text {cen }}>0.7$ and $M_{*}<2.6 \times 10^{12} h^{-1} M_{\odot}$ for both subsamples.

[35] S. D. Landy and A. S. Szalay, Astrophys. J. 412, 64 (1993).

[36] J. P. Dietrich, Y. Zhang, J. Song, C. P. Davis, T. A. McKay, L. Baruah, M. Becker, C. Benoist, M. Busha, L. A. N. da Costa, J. Hao, M. A. G. Maia, C. J. Miller, R. Ogando, A. K. Romer, E. Rozo, E. Rykoff, and R. Wechsler, Mon. Not. R. Astron. Soc. 443, 1713 (2014).

[37] M. R. Becker and A. V. Kravtsov, Astrophys. J. 740, 25 (2011).

[38] J. D. Cohn, A. E. Evrard, M. White, D. Croton, and E. Ellingson, Mon. Not. R. Astron. Soc. 382, 1738 (2007). 